\title{
Die Covid-19-Pandemie: ein massiver Stress-Test für uns alle
}

\section{Coronavirus (COVID-19) pandemic: a massive stress test for all of us}

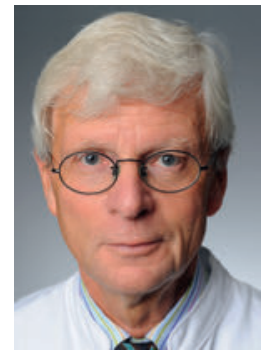

Prof. Dr. med. Joachim Klosterkötter
Korrespondenzadresse

Prof. Dr. med Joachim Klosterkötter

Klinik und Poliklinik für Psychiatrie und Psychotherapie,

Universität Köln

Kerpener Str. 62,

50924 Köln

E-Mail: joachim.klosterkoetter@uk-koeln.de

Bibliografie

DOI https://doi.org/10.1055/a-1130-7166

Fortschr Neurol Psychiatr 2020; 88: 360-361

(c) Georg Thieme Verlag KG Stuttgart · New York

ISSN 0720-4299
Was macht die Pandemie mit unserer Seele?

Diese Frage wird unter dem Eindruck der gravierenden gesamtgesellschaftlichen Krisensituation, die Covid-19 weltweit hervorgerufen hat, inzwischen natürlich immer wieder gestellt.

Was bedeutet es für die seelische Gesundheit, wenn der „Hoffnungspegel“ der Bevölkerung nach den jüngsten demoskopischen Erhebungen in Deutschland soweit abgesunken ist wie seit dem katastrophalen Ende des 2. Weltkriegs nicht? Muss nicht allein schon die tägliche Konfrontation mit der ubiquitären Ansteckungsgefahr und den möglichen Erkrankungs- oder sogar Todesfolgen Angst und Schrecken verbreiten? Werden die drastischen Einschränkungen des sozialen Lebens nicht, je länger sie andauern, immer mehr auf die Stimmung drücken und über Wut und Frust schließlich zu Depressionen führen? Drohen nicht insgesamt dieselben Belastungsreaktionen und seelischen Traumatisierungen, wie wir sie von lebensgefährlichen Großereignissen wie Kriegen oder Naturkatastrophen kennen?

Um diese Fragen zutreffend beantworten zu können, macht es sehr viel Sinn, die gegenwärtige virale Pandemie auch einmal aus dem Blickwinkel der modernen Stressforschung [1] zu betrachten. So gesehen stellt sie dann nämlich in der Tat gar nichts anderes als einen groß angelegten, der Menschheit von außen aufgezwungenen Stress-Test dar, dem wir alle einzeln und gemeinsam gleichermaßen unterworfen sind.

Wie man reagiert, hängt weniger von den objektiven Gegebenheiten der Gefährdung und Einschränkung im jeweiligen eigenen Lebensbereich, als vielmehr von deren subjektiver Bewertung ab. Wer beispielsweise seine täglichen Belastungen durch die virale Krise mehr als Herausforderung erlebt, die er unter eigener „internaler“ Kontrolle halten und selber meistern kann, wird weniger mit Ängsten oder depressiven Verstimmungen reagieren. Wenn ich mich dagegen etwa Quarantäne-Maßnahmen wie einem von außen aufgezwungenen „externalen” Schicksal ausgeliefert fühle, erhöht sich mein Risiko, auf diese Belastungen immer mehr mit „toxischem“, bei langfristiger oder hochfrequenter Exposition auch tatsächlich krankmachendem Stress zu reagieren [2].

„The Lancet“ hat kürzlich eine Studie zu den Auswirkungen häuslicher Quarantäne auf die Psyche veröffentlicht, in die Erfahrungen von insgesamt 20.000 Betroffenen eingeflossen sind. Die Ergebnisse bestätigen noch einmal eindrucksvoll, wie sehr es tatsächlich auf die eigene kognitive Bewertung der Stress-induzierenden Maßnahmen ankommt. Je mehr ich über den Sinn der Quarantäne aufgeklärt bin, mir dementsprechend auch selbst ihre Zielsetzung zu eigen machen und die Maßnahme gewissermaßen als „altruistisch“ motivierte Selbstisolierung verstehen kann, umso eher bleibe ich von den in der Studie nachgewiesenen posttraumatischen Stresssymptomen verschont [3].

Gut, schnell, aktuell und vor allem richtig informiert zu sein und dies genauso auch über die ganze Krisenentwicklung hinweg zu bleiben, ist also von ausschlaggebender Bedeutung. Deshalb kommt ja dem viel beschworenen Krisenmanagement auf allen politischen Ebenen und auf allen gesellschaftlichen Feldern tatsächlich auch ein so hohes Maß an Verantwortung für die Akzeptanz der gesundheitlichen und wirtschaftlichen Belastungen zu. Optimale Information und Überzeugungsarbeit im medial vermittelten Zusammenspiel von Experten und Politik können und sollen Einstellungen befördern, aus denen heraus man sich auch schwerwiegende Einschränkungen zu eigenen Gestaltungsaufgaben macht. Wem es gelingt, diese innere Haltung einzunehmen 
und auch unter zunehmender Belastung weiter beizubehalten, der wird wie von selbst auch zu allen weiteren aktiven Anpassungsleistungen tendieren, die für die Erhaltung seelischer Gesundheit empfehlenswert sind. Vor allem Aktivitäten wie den Alltag positiv umzugestalten, sich weiter auszutauschen und einander zu helfen, gehören zu diesen stressvermindernden Selbstregulationen mit hinzu [4].

Allerdings werden erst längerfristige kontinuierliche Erkundungen des Schutzverhaltens der Bevölkerung vor SARS-CoV-2, wie sie in Deutschland etwa das sogenannte COSMO-Konsortium betreibt, Auskunft über das Gelingen oder Scheitern der Stressbewältigung geben können [5]. Je länger die Krise andauert, umso mehr kommen ja zu der gesundheitlichen Gefährdung auch noch Belastungen durch häusliche Überforderung, Gewaltausbrüche, Vereinsamung oder drohende wirtschaftliche Notlagen hinzu. Wer hierauf schließlich mehr und mehr mit Gefühlen des Ausgeliefertseins reagiert, gerät leicht in eine abgeschlossene Welt von sich steigernden Sorgen hinein, in der dann in der Tat auch ein erhöhtes Risiko für die Entwicklung von Angsterkrankungen, depressiven Störungen und Suizidalität besteht.

Die Herstellung und fortgesetzte Förderung einer eigenverantwortlichen Umgangsmöglichkeit mit der Krise in der jeweils ganz konkreten Lebenswelt aller mehr oder weniger Stress-empfindlichen und resilienten Bevölkerungsgruppen ist also sicher das Wichtigste. Man tut aber gut daran, sie schon vorsorglich auch durch die Schaffung von Beratungs-, Betreuungs- und Hilfsangeboten für aus eigener Kraft nicht mehr zu bewältigende StressSituationen zu ergänzen. In diesen, so auch in der 3. Ad-hoc-Stellungnahme der Leopoldina zur Corona-Virus-Pandemie [6] geforderten Anlaufstellen kommt es dann aber letztlich auch sehr stark auf präsenten oder zumindest abrufbaren psychiatrischen Sachverstand an. Denn es geht ja nicht nur um die Beherrschung der jeweiligen Notsituationen, sondern vor allem auch um die beiden zentralen Aufgaben präventiver Psychiatrie: Risiken für psychische Neu- oder Wiedererkrankungen früh zu erkennen und die drohenden Angsterkrankungen, Depressionen und anderen psychischen Störungen durch geeignete Risiko-adaptierte Maßnahmen wie Familienberatung, Stressbewältigung und ResilienzAufbau rechtzeitig zu verhindern.
Kommen wir also auf die Ausgangsfrage, was die Pandemie mit unserer Seele macht, zurück. Sie setzt sie zweifellos wie ein massiver Stress-Test unter einen außerordentlich hohen Belastungsdruck und mindert dementsprechend weitgehend auch unser psychisches Wohlbefinden. Zu einer Zunahme psychischer Neu- oder Wiedererkrankungen muss es jedoch auch unter diesen dramatischen Umständen nicht zwingend kommen. Aufklärung und informiertes Verständnis können selbstregulative Kräfte aktivieren und auch stressempfindliche Menschen zu ganz ungewöhnlichen Anpassungsleistungen anregen. Außerdem hat sich das psychiatrische und psychotherapeutische Angebot zur Früherkennung von krankmachendem Stress und zum Abfangen stressassoziierter Erkrankungen in den letzten 20 Jahren in Deutschland deutlich verbessert.

\section{Literatur}

[1] Klosterkötter J, Maier W. Konzeptionelle Grundlagen der Krankheitsentwicklung und Resilienz bei psychischen Störungen - Präventionsansätze. In: Klosterkötter J, Maier W (Hrsg.) Handbuch Präventive Psychiatrie. Forschung-Lehre-Versorgung. Stuttgart: Schattauer 2017, S. 43-101

[2] Karatsoreos IN, McEwen BS. Psychobiological allostasis, resistance, resilience and vulnerability. Trends Cogn Sci 2011; 15:576-84. doi: 10.1016/j.tics.2011.10.005

[3] Brooks SK, Webster RK, Smith LE et al., The psychological impact of quarantine and how to reduce it: rapid review of the evidence, Lancet 2020; 395: 912-20. https://doi.org/10.1016/S0140-6736(20)30460-8

[4] DGPPN (Deutsche Gesellschaft für Psychiatrie und Psychotherapie, Psychosomatik und Nervenheilkunde). Pressemitteilung Coronavirus: Tipps für die seelische Gesundheit vom 20.03.2020 (https://dgppn.de/schwe rpunkte/corona-psyche.html)

[5] COVID-19 Snapshot Monitoring (COSMO) http://dx.doi.org/10.23668/ psycharchives.2776) Leopoldina Nationale Akademie der Wissenschaften. Dritte Ad-hoc-Stellungnahme vom 13.04.2020: Coronavirus-Pandemie - Die Krise nachhaltig überwinden. (https://www.leopoldina.org/ uploads/tx_leopublication/2020_04_13_Coronavirus-Pandemie-Die_Kri se_nachhaltig_\%C3\%BCberwinden_final.pdf)

[6] Leopoldina Nationale Akademie der Wissenschaften. Dritte Ad-hocStellungnahme vom 13.04.2020: Coronavirus-Pandemie - Die Krise nachhaltig überwinden. (https://www.leopoldina.org/uploads/tx_leopublication/2020_04_13_Coronavirus-Pandemie-Die_Krise_nachhaltig_\%C3\%BCberwinden_final.pdf) 